\title{
ESVAZIAMENTO GÁSTRICO NOS PACIENTES COM INSUFICIÊNCIA RENAL CRÔNICA SUBMETIDOS À HEMODIÁLISE
}

\section{Gastric emptying study in patients with chronic renal failure on hemodialysis}

\author{
Eunice Sizue HIRATA, Maria Aparecida MESQUITA, Edwaldo Eduardo CAMARGO, \\ Cecília Hirata TERRA, Gentil ALVES-FILHO
}

ABCDDV/607

Hirata ES, Mesquita MA, Camargo EE, Terra CH, Alves-Filho G. Esvaziamento gástrico nos pacientes com insuficiência renal crônica submetidos à hemodiálise. ABCD Arq Bras Cir Dig 2008;21(3):120-3

RESUMO - Racional - Queixas dispépticas são comuns em pacientes com insuficiência renal crônica. As mais frequentemente relatadas são anorexia, náusea, vômito, sensação de plenitude gástrica e dor epigástrica. A possibilidade destes sinais e sintomas estarem associadas ao retardo no esvaziamento gástrico é atraente. Objetivo - Estudar o esvaziamento gástrico de uma refeição sólida padronizada, em pacientes com insuficiência renal crônica em tratamento dialítico. Método - Foram estudados 31 pessoas de ambos os sexos com idade variável de 18 à 60 anos, sendo 14 com insuficiência renal crônica em hemodiálise há mais de 6 meses e 17 sadios. Foram excluídos pacientes com diabetes mellitus, amiloidose, doenças do colágeno, doenças dispépticas e/ou submetidos à operação gástrica, pacientes em uso de drogas pró-cinéticas gástricas e grávidas. O método do esvaziamento gástrico foi a cintilografia, através de câmara de cintilação de dois cabeçotes. A refeição teste padronizada consistiu de omelete de três ovos de galinha preparado com mistura de enxofre coloidal marcado com $185 \mathrm{MBq}$ de tecnécio-99m. Foram estudados a curva de retenção gástrica total e o $\mathrm{T}^{1} / 2$ do esvaziamento gástrico. Os testes estatísticos utilizados foram o de c 2 e o de Kruskal Wallis. Resultados - Os resultados confirmaram a homogeneidade dos grupos quanto à idade e o sexo. Não houve diferença estatisticamente significativa em relação às curvas de retenção gástrica total e o T1/2, semelhantes nos dois grupos. Conclusão - O esvaziamento gástrico de pacientes urêmicos em tratamento hemodialítico há mais de seis meses é igual ao de indivíduos sadios.

DESCRITORES - Insuficiência renal crônica. Diálise renal. Esvaziamento gástrico.

\section{INTRODUÇÃO}

Queixas dispépticas são comuns em pacientes com insuficiência renal crônica (IRC). As mais frequentemente relatadas são anorexia, náusea, vômito, sensação de plenitude gástrica e dor epigástrica ${ }^{20}$. A possibilidade destes sinais e sintomas estarem associadas a retardo no esvaziamento gástrico (EG) é atraente ${ }^{8}$ e não faltam, no paciente urêmico, causas para explicar esta disfunção: acidose metabólica, anemia ${ }^{16}$ e neuropatia ${ }^{2}$. Desde a observação de Goldstein, de retardo no EG em urêmicos em 1986, vários trabalhos têm apresentado resultados bastante conflitantes 5 . Falta de padronização das refeições testes, da gravidade e do tipo de tratamento da IRC e do método de estudo do EG poderiam explicar os diferentes resultados encontrados na literatura.

A importância do EG em anestesia pode ser resumida em um de seus aspectos principais: o jejum pós-operatório. Retardo pode causar estase gástrica, predispondo ao risco de vômito e aspiração ${ }^{12}$. A importância clínica pode ser entendida se nos detivermos ao grande enfoque que se

Trabalho realizado no Hospital de Clínicas da Faculdade de Ciências Médicas da Universidade Estadual de Campinas - UNICAMP, Campinas, SP, Brasil

Correspondência:Eunice Sizue Hirata, e-mail: eshirata@hotmail.com tem dado aos transplantes renais, como principal forma de tratamento da IRC. Medidas especiais na indução e recuperação da anestesia são recomendadas, com o intuito de evitar as graves complicações pulmonares que podem ocorrer nas situações de distúrbios do $\mathrm{EG}^{7}$.

O objetivo deste estudo foi estudar o EG em pacientes com IRC em tratamento hemodialítico e compará-lo a um grupo de individuo sadios, utilizando-se o método da cintilografia gástrica.

\section{MÉTODO}

Este trabalho foi aprovado pelo Comitê de Ética em Pesquisa da Faculdade de Ciências Médicas da Unicamp com o termo de consentimento tendo sido assinado na entrevista, quando o paciente era convidado a participar da pesquisa, após esclarecimentos sobre o estudo

Foram estudados 14 pacientes com IRC em tratamento hemodialítico há mais de seis meses no Hospital das Clínicas da Unicamp (Grupo THD). Os critérios de exclusão foram presença de diabete melito, amiloidose, doenças do colágeno, úlcera péptica, cirurgia gástrica prévia e gravidez. Nenhum deles estava em uso de medicações que pudessem afetar a motilidade gastrintestinal.

O grupo-controle (CTL) foi constituído por 18 vol- 
untários assintomáticos: nove do sexo masculino (idade média: $35 \pm 9$ anos) e nove do feminino (idade média: 41 \pm 11 anos), recrutados entre funcionários e médicos do HCUNICAMP. Nenhum participante era obeso, ou apresentava história de doença sistêmica, gastrintestinal, nenhum foi submetido à cirurgia prévia ou estava em uso de medicações que pudessem afetar a motilidade gastrintestinal. Nas voluntárias do sexo feminino, os estudos foram realizados durante a primeira fase do ciclo menstrual.

A refeição teste consistiu de uma omelete com três ovos de galinha tamanho médio preparada com uma mistura de enxofre coloidal marcado com $185 \mathrm{MBq}$ de tecnécio-99m. A omelete foi cozida em forno de microondas por quatro minutos, até adquirir consistência firme.

No estudo do esvaziamento gástrico os exames foram realizados de acordo com a técnica já publicada anteriormente $^{14}$. Os participantes foram estudados pela manhã, após jejum de pelo menos 10 horas. A refeição-teste foi consumida em cinco minutos e foi seguida da ingestão de $25 \mathrm{~mL}$ de água. Imediatamente após a ingestão, o indivíduo era colocado sentado, entre os dois cabeçotes da câmara de cintilação, para a aquisição de imagens nas projeções anterior e posterior da região epigástrica, que foi colocada no centro do campo de visão dos dois cabeçotes.

Foram adquiridas imagens estáticas do estômago nas incidências anterior e posterior simultaneamente. A partir do instante zero, imediatamente após a ingestão da refeição, e a cada 10 minutos, obteve-se uma imagem de 60 segundos de duração, até se completar 120 minutos, num total de 26 imagens, 13 na posição anterior e 13 na posterior. As imagens foram processadas através do programa SHOPHY NXT do Serviço de Medicina Nuclear. Inicialmente foram determinadas 26 regiões de interesse de estudo nas projeções anterior e posterior. Após correção para o decaimento radioativo, foi determinada a média geométrica das contagens radioativas da região anterior e posterior. Obtiveram-se 13 valores de média geométrica, normatizadas pelo maior valor em escala de zero a um. Com estes dados foram construídos os gráficos de retenção gástrica total, contendo na abscissa o tempo em minutos e na ordenada a contagem da radioatividade. A partir da curva de retenção gástrica total foi calculado o $T^{1} 12$ do $E G$ total, tempo que o estômago requer para esvaziar $50 \%$ da refeição teste padronizada.

O tratamento estatístico constou do teste do c2 para comparação de variáveis qualitativas e de Kruskal-Wallis para comparar três ou mais conjuntos de informações numéricas de amostras independentes. Foram considerados estatisticamente significativos, valores de $P$ menores que 0,05 .

Para a análise individual dos resultados, o intervalo de normalidade dos valores do $T^{1} / 2$ do EG foi considerado como a média $\pm 2 \mathrm{DP}$ dos valores de $\mathrm{T}^{1} 1 / 2$ observados no grupo-controle.

\section{RESULTADOS}

A análise dos dados confirmou a homogeneidade dos grupos para idade e sexo (Tabela 1). O tempo médio de hemodiálise foi de $32.9 \pm 40.2$ meses.
TABELA 1 - Distribuição por sexo e idade média (M), desviopadrão (DP), valor mínimo e máximo (Min-Max) em anos nos grupos THD e CTL

\begin{tabular}{cll}
\hline & Grupo THD & Grupo CTL \\
\hline *Idade M + DP & $42.5 \pm 11.2$ & $36.6 \pm 10.6$ \\
Min-Max & $18-60$ & $24-55$ \\
$* *$ Sexo M / F & $6 / 8$ & $9 / 8$ \\
\hline
\end{tabular}

* Kruskal-Wallis: $P=0.353$

**c2: $P=0.751$

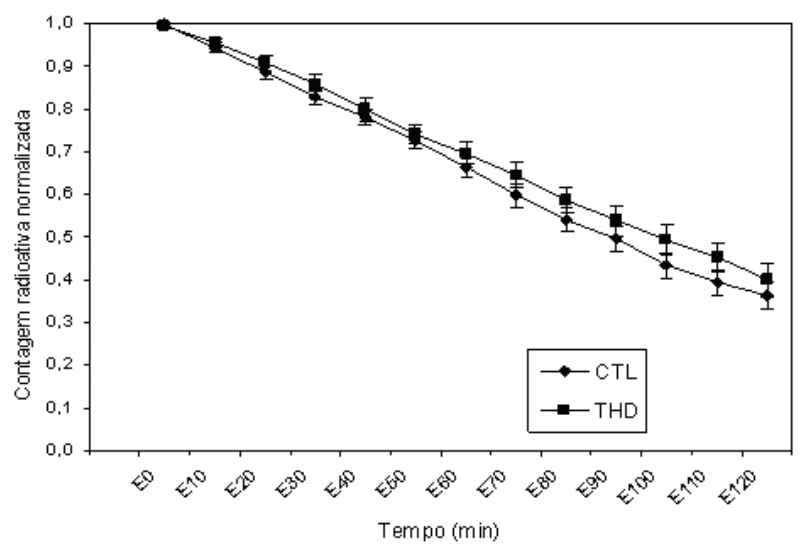

FIGURA 1 - Representação gráfica da média e erro padrão dos valores da contagem radioativa normalizada $\mathrm{RG}$ total versus tempo em minutos, em pacientes com IRC em hemodiálise (THD) e em indivíduos sadios (CTL).

Não houve diferença estatisticamente significativa entre os grupos nos tempos estudados. Os valores de $\mathrm{T}^{1} / 2$ de EG podem ser vistos na Tabela 2

TABELA 2 - Valores da média, desvio padrão, mínimo e máximo do $\mathrm{T}^{1} \frac{1}{2}$ de EG em pacientes dos Grupos THD e CTL

\begin{tabular}{lll}
\hline \multicolumn{1}{c}{$\mathbf{T} 12$ (min) } & Grupo THD & Grupo CTL \\
\hline Média+DP & $102.9 \pm 31.2$ & $87.2 \pm 18.9$ \\
Min-Max & $64.7-172.7$ & $67.3-132.7$ \\
$\mathrm{~N}$ & 14 & 17 \\
\hline
\end{tabular}

Kruskal-Wallis: $P=0.176$

\section{DISCUSSÃO}

Desde o primeiro registro de retardo no EG em urêmicos, observa-se que as publicações apresentam resultados conflitantes. A maioria destes trabalhos são estudos clínicos, onde percebe-se falhas na padronização não apenas do método empregado para estudo do EG como também nas refeições testes utilizadas, na classificação do grau de disfunção renal e no tipo de tratamento de substituição renal a que o paciente está sendo submetido ${ }^{6}$. Também são poucas as referências quanto à exclusão de doenças gástricas concomitantes e de sistêmicas que evoluem com retardo no EG. Estas variáveis já seriam suficientes para justificar os resultados paradoxais encontrados na literatura.

Neste estudo procurou-se controlar as variáveis que 
poderiam influir na taxa de EG. Começou-se pelo padrão de normalidade adotado neste estudo, que foi obtido a partir de uma refeição teste em um grupo de indivíduos sadios. Em relação à idade e ao sexo, definiu-se para os dois grupos estudados que a idade deveria estar compreendida entre 18 e 60 anos e cuidou-se para que a distribuição por sexo fosse semelhante nos dois grupos. Embora existam controvérsias, parece que o EG é mais rápido em indivíduos do sexo masculino, porque os esteróides teriam papel inibidor na motilidade gástrica ${ }^{14}$. Com a menopausa este efeito tenderia a desaparecer e as taxas de EG a se igualar ${ }^{11}$. O efeito da idade no EG tem sido pouco estudado e os resultados não são uniformes. Entretanto, como existem relatos de comprometimento do EG em pacientes acima de 60 anos, optou-se por exclui-los do trabalho ${ }^{13}$.

Diabete melito e outras doenças, como algumas colagenoses, podem cursar com retardo no EG. A gastroparesia diabética é complicação associada ao diabete insulinodependente de longa duração, com disfunção autonômica e neuropatia periférica. Em geral estão comprometidos o esvaziamento de sólidos e de líquidos ${ }^{9}$. Já no caso das colagenoses, as mais frequentemente associadas ao retardo no EG são a esclerose sistêmica progressiva ${ }^{3} \mathrm{e} o$ lupus eritematoso sistêmico ${ }^{15}$. Mais recentemente, a amiloidose tem sido relacionada à uremia com retardo no $\mathrm{EG}^{17}$. Todos os pacientes com estas doenças foram excluídos do presente trabalho.

O método utilizado para o estudo do EG foi o da cintilografia gástrica empregando-se uma refeição sólida acrescida de um radiofármaco ${ }^{16}$. Os parâmetros utilizados foram o $\mathrm{T}^{1} / 2$ e a curva de EG total. Selecionou-se um grupo de pacientes renais crônicos em diálise há no mínimo seis meses. Segundo dados da literatura, alterações anatômicas gastrintestinais são menos frequentes em urêmicos em diálise, assim como alguns sintomas dispépticos como a náusea e o vômito, sugerindo que a diálise diminuiria a ocorrência de alguns sinais e/ou sintomas da uremia como o retardo no $\mathrm{EG}^{1}$. Os estudos do $\mathrm{EG}$ em pacientes submetidos aos diferentes tipos de tratamento de substituição renal também mostram resultados conflitantes. Guz et al. ${ }^{6}$ constataram retardo em pacientes em hemodiálise, em diálise peritoneal e em pacientes submetidos ao transplante renal, quando comparados a um grupo de indivíduos sadios. Entretanto, não encontrou diferenças no tempo de EG entre os três grupos estudados ${ }^{6}$.

A relação entre o retardo no $\mathrm{EG}$ e a diálise peritoneal e o volume e a composição do líquido de diálise tem sido objeto de muitos estudos clínicos ${ }^{18}$. Existe consenso quanto a existência de retardo em pacientes com IRC em diálise peritoneal ${ }^{4,19,10} \mathrm{e}$ de que este retardo não está relacionado ao volume do líquido infundido 18 mas à absorção de substâncias presentes no líquido de diálise ${ }^{21}$.

Neste trabalho, selecionou-se um grupo de pacientes com IRC em tratamento hemodialítico há mais de seis meses, Constatou-se que neste grupo, o EG foi igual ao de indivíduos sadios. Outro estudo futuro em pacientes com IRC terminal em tratamento clínico conservador, poderá ser esclarecedor no sentido de identificar se o tratamento por hemodiálise interfere favoravelmente na taxa de EG.

\section{CONCLUSÃo}

Não houve diferença na curva de retenção gástrica total e no $T^{1 / 2}$ de esvaziamento gástrico entre pacientes com insuficiência renal crônica e indivíduos sadios.

Hirata ES, Mesquita MA, Camargo EE, Terra CH, Alves-Filho G. Gastric emptying study in patients with chronic renal failure on hemodialysis. ABCD Arq Bras Cir Dig 2008;21(3):120-3

ABSTRACT - Background - Dyspeptic symptoms are frequent in renal patients. They are anorexia, nausea, vomit, pain and epigastric distension. These symptoms can be related to the delay in gastric emptying. Aim - To investigate gastric emptying in patients with chronic renal failure on hemodialysis treatment. Method - Thirty one subjects (18-60 y) were studied, 14 on hemodialysis for at least 6 months and 17 healthy subjects). Patients with diabetes, amyloidosis, collagenous disease, pregnant, dyspeptic complaints, gastric surgery and patients taking procinetics drugs were excluded. Gastric emptying was investigated with radionuclide scintigraphy, using a dual-head scintillation camera. The standard test meal consisted of $185 \mathrm{MBq}$ of technetium-99m sulfur colloid mixed with three scrambled eggs. For each study group a time-activity curve of gastric retention was generated and from that the $\mathrm{T}^{1} / 2$ of gastric emptying was obtained. Results - The groups were similar both in gender and age. There was no significant statistical difference in total retention curves between the two studied groups. Also the $\mathrm{T}^{1} 12$ of gastric emptying was similar in the two groups. Conclusion - Gastric emptying of patients on hemodialysis treatment for at least 6 months was not different from the health subjects.

HEADINGS - Renal insufficiency, chronic. Renal dialysis. Gastric emptying.

\section{REFERÊNCIAS}

1. Adachi H, Kamiya T, Hirako M, Misu N, Kobayashi Y, Shikano M, Matsuhisa E, Kataoka H, Sasaki M, Ohara H, Nakao H, Orito E, Joh T. J Smooth Muscle Res. 2007; 43(5): 179-89.

2. Dimitrascu DL, Barnet J, Kirschner T, Wienbeck M. - Astral emptying of semisolid meal measured by real-time ultrasonography in chronic renal failure. Dig Dis Sci. 1995; 40:636-44.

3. Franck-Larsson K, Hedenstrom H, Dahl R, Ronnblom A. Delayed gastric emptying in patients with diffuse versus limited systemic sclerosis unrelated to gastrointestinal symptoms and myoelectric gastric activity. Scand J Rheumatol. 2003; 32(6): 348-55

4. Fernstrom A, hylander B, Grybavk P, Jacobsson H, Hellstrom PM. Gastric emptying and alectrogastrography in patients on CAPD. Perit Dial Int 1999; 19(5): 429-37.
5. Goldstein H, Murphy D, Sokol A, Rubini ME. - Gastric acid secretion in patients undergoing chronic dialysis. Arch. Intern. Med. 1967; 120: 645-53.

6. Guz G, Bali M, Poyraz NY, Bagdatoglu O, Yegin ZA, Dogan I, Atasever T, Sert S, Sindel S. Gastric emptying in patients on renal replacement theraphy. Ren Fail. 2004: 26(6): 619-24.

7. Hadjiyannakis EJ, Evans DB, Smellie WAB, Calne RY. - Gastrointestinal complication following renal transplantation. Lancet 1971; 2: 781-5.

8. Hirako M, Misu N, Kobayashi Y, Adachi H, Shikano M, Matsuhisa E, Kimura G. Impaired gastric motility and its relationship to gastrointestinal syntoms in patients with chronic renal failure. J Gastroenterol. 2005; 40(12): 1116-22.

9. Horowitz M, O’Donovan D, Jone KL, Feinle C, Royner CK, Samsom M. Gastric emptying in diabetes: clinical significance and treatment. Diabet Med. 2002; 19(3): 177-94 
10. Hubalewska A, Stompor T, Placzkiewicz E, Staszczak A, Huszno B, Sulowicz W, Szybinski Z. Evaluation of gastric emptying in patients with chronic renal failure on continuous ambulatory peritoneal dialysis using $99 \mathrm{mTc}$-solid meal. Nucl Med Rev Cent East Eur. 2004; 7(1): 27-30.

11. Hutson WR, Roehrkasse RL, Wald A. - Influence of gender and menopause on gastric emptying and motility. Gastroenterology 1989; 96:11-7.

12. Kallar SK, Everett LL. - Potencial risks and preventive measures for pulmonary aspiration: new concepts in preoperative measures for pulmonary aspiration: new comcepts in preoperative fasting guidelines. Anesth. Analg. 1993; 77; 171-82.

13. Kao CH, Lai TL, Wang SJ, Chen GH, Yet SH. - Influence of age on gastric emptying in healthy chinese. Clin. Nucl. Med. 1994; 19: 401-4.

14. Lorena SLS, Tinois E, Hirata ES, Cunha ML, Brunetto SQ, Camargo EE, Mesquita MA. - Estudo do esvaziamneto gástrico e da distribuição intragástrica de uma dieta sólida através da cintilografia: diferença entre sexos. Arq Gastroenterol. 2000; 37(2):102-6.

15. Minami H, McCallum RW. - The physiology and pathophysiology of gastric emptying in humans. Gastroenterology 1984; 86:1592-610.

16. Ravelli AM.- Gastrointestinal function in chronic renal failure. Pediatr. Nephrol. 1995; 9: 756-62
17. Sglam F, Celik A, Cavdar C, Sifil A, Atila K, Kaya GC, Bora S, Gulay H, Camsari T. A renal transplant recipient with delayed gastric emptying in amyloidosis due to familial Mediterranean fever improved with erythromycin: a case report. Transplant Proc. 2008; 40(1): 308-9.

18. Schoonjans R, Van Vlem B, Vandamme W, Van Vlierberghe H, Van Heddeghem N, Van Biesen W, Mast A, Sas S, Vanholder R, Lameire N, De Vos M. Gastric emptying of solids in cirrhotic and peritoneal dialysis patients: influence of peritoneal volume load. Eur J Gastroenterol Hepatol. 2002; 14(4); 395-8

19. Strid H, Simren M, Stotzer PO, Abrahamsson H, Bjornsson ES. Delay in gastric emptying in patients with chronic renal failure. Scand J Gastroenterol. 2004; 39(6): 516-20.

20. Van Vlen B, Schoonjans R, Vanholder R, Vandamme W, De Vos M, Lameire N. Dyspepsia and gastric emptying in chronic renal failure patients. Clin Nephrol. 2001; 56(4): 302-7.

21. Van V, Schoonjans RS, Strujik DG, Verbanck JJ, Vanholder RC, Van B, Lefebve RA, De V, Lameire NH. Influence of dialysate on gastric emptying time in peritonel dialysis patients. Perit Dial Int. 2002; 22(1): 32-8.

Fonte de financiamento: não há Conflito de interesse: não há Recebido para publicação: 03/02/2008 Aceito para publicação: 05/05/2008 